\title{
Multi-Scale Examination of the Effect of Grain Boundary Character on Irradiation-Induced Defects: A Comparison of In Situ and Ex Situ Techniques
}

\author{
C.M. Barr,* G. Vetterick,* K. Hattar,** M.A. Kirk,*** A. Misra****, and M.L. Taheri* \\ * Department of Materials Science and Engineering, Drexel University, Philadelphia, PA 19104 \\ **Radiation Solid Interactions, Sandia National Laboratories, Albuquerque, NM 87185 \\ *** Materials Science Division, Argonne National Laboratory, Argonne, IL 60439 \\ **** Materials Physics and Applications Division, Los Alamos National Laboratory, Los Alamos, \\ New Mexico 87545
}

Advanced nuclear energy systems will require materials to perform for extended periods under conditions of elevated temperatures and extreme radiation. Conventional materials (fcc Ni- and Fe-based alloys) lack the required microstructural stability and will exhibit excessive coarsening, hardening, and swelling. Grain boundaries in metallic materials can substantially reduce this degradation by acting as highly effective sinks for point defects [1]. One potential solution to obtain microstructures that are more stable under irradiation is grain boundary engineering. Grain boundary engineering is associated with specific thermomechanical processing routes to yield a specific fraction of annealing twins and twin variant boundaries $\left(\Sigma 3^{\mathrm{n}}, \mathrm{n}=1-3\right)$. Grain boundary networks that have consisted of high fractions of these boundaries have previously been shown to improve grain boundary mediated degradation including creep, corrosion and hydrogen embrittlement [1-2]. In order to develop a microstructure that is more radiation tolerant, the interaction between specific twin related grain boundaries and irradiation induced point defects is necessary.

In this study, we employ an experimental method to combine multi-length scale quantitative microstructural characterization to study the interaction between specific twin related grain boundaries and irradiation induced defects. First, a strain-annealed thermomechanical grain boundary engineering process was deployed on 316L stainless steel as method to increase the fraction of $\Sigma 3^{\mathrm{n}}(\mathrm{n}=1,2,3)$ coincidence site lattice (CSL) grain boundaries. The processing route, as indicated by electron backscatter diffraction (EBSD) grain boundary character map in Figure 1, increased the length fraction of twin related boundaries to $65 \%$. The $316 \mathrm{~L}$ grain boundary engineered samples, as characterized by orientation microscopy, where then ion-irradiated at specific locations that had a high density of the special low-CSL boundaries. Following irradiation, we employ a site-specific sample preparation technique to investigate the interaction of irradiation induced defects with twin-related boundaries by high-resolution transmission electron microscopy (HRTEM). We will present results that discuss the interaction of the low free volume CSL boundaries and the irradiation induced point defects.

In addition to the ex-situ irradiation and TEM characterization, in-situ TEM ion beam irradiation and heating experiments will be presented for the 316L grain boundary engineered samples. The in-situ ion beam irradiation provides the ability to study the role grain boundary character and the changing behavior of the irradiation induced defects during radiation (Figure 2 indicates preliminary results). The results of the TEM studies will be correlated with meso-scale in-situ scanning electron microscopy tensile and EBSD experiments. The coupling of EBSD and tensile testing enables realtime analysis of grain boundary character during straining. The multi-scale characterization techniques used in the present study will help provide a connection of the role of twin and twin-variant grain boundaries under irradiation. 


\section{References}

[1] S. Bechtle et. al., Acta Materialia. 57(14), (2009) 4148-4157.

[2] B.W. Reed et. al., Acta Materialia, 56(13), (2008) 3278-3289.

[3] This research was made possible by use of the Centralized Research Facilities in the College of Engineering at Drexel University. This research was supported by the U.S. DOE by the EMC for Materials Research at ANL, a U.S. DOE Office of Science Laboratory operated under Contract No. DE-AC02-06CH11357 by UChicago Argonne, LLC, in part by the US DOE by Sandia National Laboratory's Ion Beam Facility, and in part by the Center for Integrated Nanotechnologies at Los Alamos National Laboratory. MT thanks the US Nuclear Regulatory Commission and the US DOE-
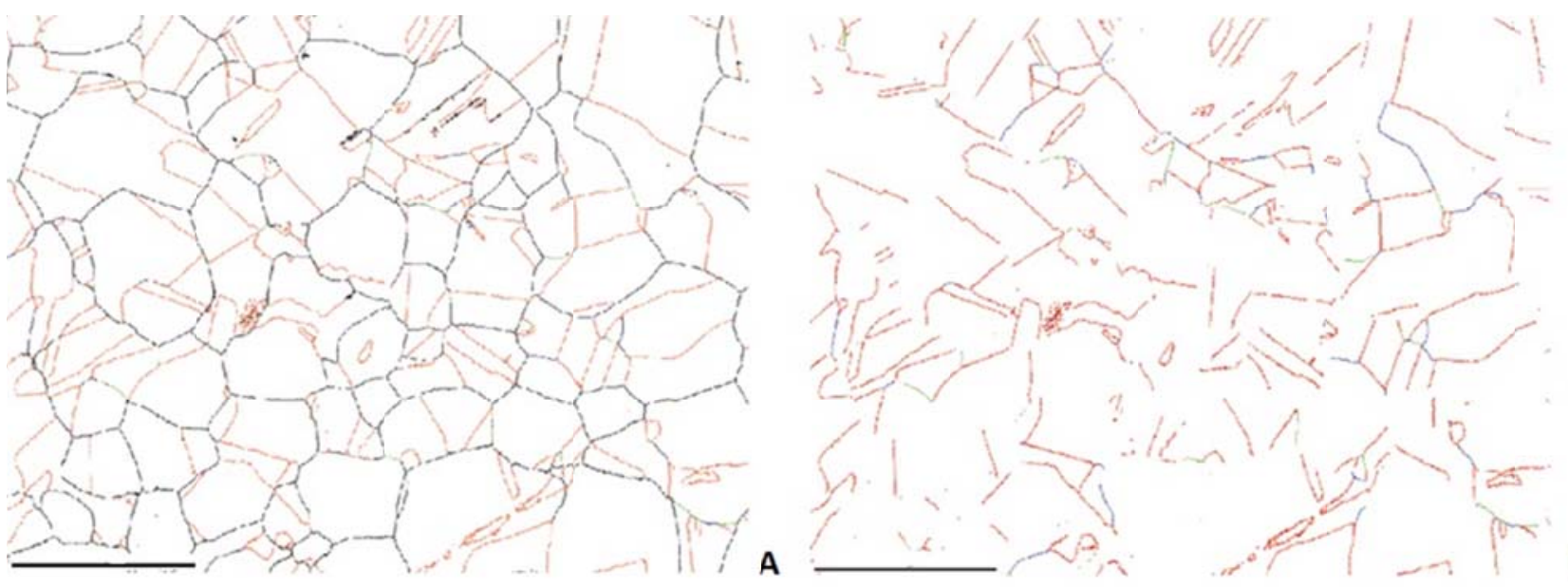

B

NEUP for funding in support of this research

Figure 1: EBSD grain boundary inverse pole figure map images of the grain boundary character distribution of $316 \mathrm{~L}$ after the grain boundary engineering thermomechanical processing. A: all boundary types; and B: only CSL-type boundaries. Scale bar $=100$ microns.
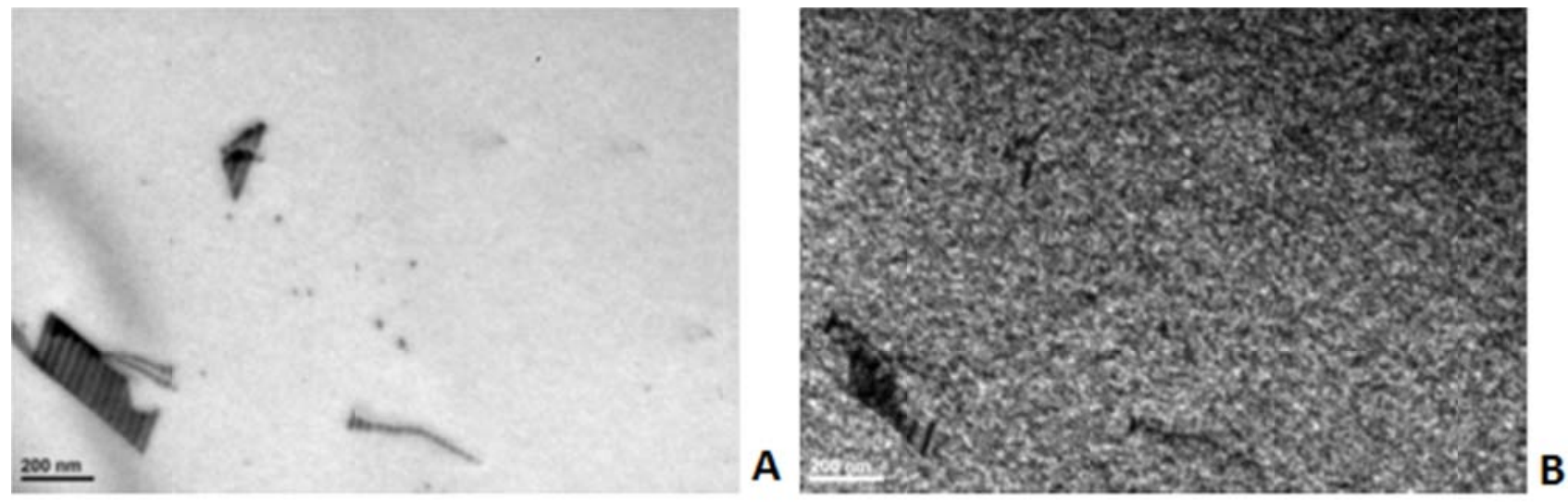

Figure 2: Bright field TEM image showing austenitic stainless steel under ion-irradiated at $180^{\circ} \mathrm{C}$. A: the location before ion-irradiation at $180^{\circ} \mathrm{C}$; and $\mathrm{B}$ : the location after .4 displacement per atom ion-irradiation at $180^{\circ} \mathrm{C}$. Small dislocation loops in B are illustrated by black and white contrast. 